\title{
UCRL-TR-214888
}

LAW RENCE LIVERMORE N A T IO N A L LABORATORY

Mark Ulitsky

August 30, 2005

The Benjamin Shock Tube Problem in KULL 
This document was prepared as an account of work sponsored by an agency of the United States Government. Neither the United States Government nor the University of California nor any of their employees, makes any warranty, express or implied, or assumes any legal liability or responsibility for the accuracy, completeness, or usefulness of any information, apparatus, product, or process disclosed, or represents that its use would not infringe privately owned rights. Reference herein to any specific commercial product, process, or service by trade name, trademark, manufacturer, or otherwise, does not necessarily constitute or imply its endorsement, recommendation, or favoring by the United States Government or the University of California. The views and opinions of authors expressed herein do not necessarily state or reflect those of the United States Government or the University of California, and shall not be used for advertising or product endorsement purposes.

This work was performed under the auspices of the U.S. Department of Energy by University of California, Lawrence Livermore National Laboratory under Contract W-7405-Eng-48. 


\section{The Benjamin Shock Tube Problem in KULL}

\section{Mark Ulitsky AX-Division L-023}

The goal of the EZturb mix model in KULL is to predict the turbulent mixing process as it evolves from Rayleigh-Taylor, Richtmyer-Meshkov, or Kelvin-Helmholtz instabilities. In this report we focus on a simple example of the Richtmyer-Meshkov instability (which occurs when a shock hits an interface between fluids of different densities) without the complication of reshock. The experiment by Benjamin et al. [1], involving a Mach 1.21 incident shock striking an air / $\mathrm{SF}_{6}$ interface, is a good one to model and understand before moving onto shock tubes that follow the growth of the turbulent mixing zone from first shock through well after reshock [2-3].

In Kull, the EZturb k- $\varepsilon$ model is tightly coupled to the Lagrange hydro, and so the actual mix model equations we solve when the model is active are:

$$
\begin{aligned}
& \frac{d \alpha_{r}}{d t}=\frac{\partial}{\partial x_{j}}\left(\frac{\mu_{t}}{\sigma_{M}} \frac{\partial}{\partial x_{j}}\left(\frac{\alpha_{r}}{\rho}\right)\right) \\
& \frac{d \alpha_{r} \rho_{r}}{d t}=\frac{\partial}{\partial x_{j}}\left(\frac{\mu_{t}}{\sigma_{M}} \frac{\partial}{\partial x_{j}}\left(\frac{\alpha_{r} \rho_{r}}{\rho}\right)\right) \\
& \frac{d \rho u_{i}}{d t}=-\frac{\partial \tau_{i j}}{\partial x_{j}} \\
& \frac{d \alpha_{r} \rho_{r} I_{r}}{d t}=\frac{\partial}{\partial x_{j}}\left(\frac{\mu_{t}}{\sigma_{U}} \frac{\partial}{\partial x_{j}}\left(\frac{\alpha_{r} \rho_{r} I_{r}}{\rho}\right)\right)+P_{r}^{I}+\delta_{I, D i s s} \alpha_{r} \rho_{r} \varepsilon \\
& \frac{d \rho k}{d t}=\frac{\partial}{\partial x_{j}}\left(\frac{\mu_{t}}{\sigma_{K}} \frac{\partial k}{\partial x_{j}}\right)-\tau_{i j} S_{i j}-\rho \varepsilon+P \\
& \frac{d \rho \varepsilon}{d t}=\frac{\partial}{\partial x_{j}}\left(\frac{\mu_{t}}{\sigma_{Z}} \frac{\partial \varepsilon}{\partial x_{j}}\right)-C_{1 \varepsilon} \tau_{i j} S_{i j} \frac{\varepsilon}{k}-C_{2 \varepsilon} \rho \frac{\varepsilon^{2}}{k}+C_{3 \varepsilon} P \frac{\varepsilon}{k}
\end{aligned}
$$

Here, $\alpha_{r}, \rho_{r}$, and $\mathrm{I}_{\mathrm{r}}$ are the volume fraction, thermodynamic density, and specific internal energy (by mass) for material $r . S_{\mathrm{ij}}$ is the strain rate tensor, and $\tau_{\mathrm{ij}}$ is the turbulent shear stress tensor, for which we use the following Boussinesq approximation: 


$$
\tau_{i j}=\delta_{I s o} \frac{2}{3} \rho k \delta_{i j}-\delta_{\text {Anso }} 2 \mu_{t}\left(S_{i j}-\frac{\delta_{i j}}{3} \frac{\partial u_{k}}{\partial x_{k}}\right)
$$

The turbulent viscosity includes the effects of both shear and buoyancy and takes the form:

$$
\mu_{t}=C_{\mu} \rho \frac{k^{2}}{\varepsilon}+C_{\omega} \rho \frac{k^{3}}{\varepsilon^{2}} \sqrt{\omega^{2}+\frac{\nabla p \cdot \nabla p}{p \rho}-\frac{\nabla p \cdot \nabla \rho}{\rho^{2}}[1-\Theta(\nabla p \cdot \nabla \rho)]}
$$

The unlimited form of the buoyant production term is given by:

$$
P=-\frac{\mu_{t}}{\sigma_{\rho} \rho^{2}} \nabla p \cdot \nabla \rho
$$

and the way this term manifests itself in the internal energy equation is:

$$
P_{r}^{I}=-h_{r} p_{r} \nabla \cdot\left(\frac{\mu_{t}}{\sigma_{\rho} \rho^{2}} \nabla \rho\right)
$$

The model constants are $\mathrm{C}_{1 \varepsilon}, \mathrm{C}_{2 \varepsilon}, \mathrm{C}_{3 \varepsilon}, \sigma_{\mathrm{M}}, \sigma_{\mathrm{U}}, \sigma_{\mathrm{K}}, \sigma_{\mathrm{Z}}, \sigma_{\rho}, \mathrm{C}_{\mu}$, and $\mathrm{C}_{\omega}$. Also, $\delta_{\text {Iso }}, \delta_{\text {Aniso }}$, and $\delta_{\mathrm{I}, \mathrm{diss}}$ are on/off switches that can be set to 1 or 0 . To simplify the form of the turbulent viscosity and the Reynolds stress, we will set $\mathrm{C}_{\omega}=0$ and $\delta_{\text {Aniso }}=0$ for this problem.

We are given that the incident Mach number is 1.21 and that the ambient densities for the air and $\mathrm{SF}_{6}$ are $.9524 \mathrm{e}-3 \mathrm{~g} / \mathrm{cm}^{3}$ and $4.8611 \mathrm{e}-3 \mathrm{~g} / \mathrm{cm}^{3}$. The ambient pressure is also given as .8 bars. With these values and the standard 1D shock relations [4-5], we can compute shocked values for the air (we assume $\gamma_{\text {air }}=1.4$ ). Thus the shocked air density, pressure, and velocity are found to be $1.294 \mathrm{e}-3 \mathrm{~g} / \mathrm{cm}^{3}, 1.233 \mathrm{e} 6 \mathrm{dynes} / \mathrm{cm}^{2}$, and $1.096 \mathrm{e} 4$ $\mathrm{cm} / \mathrm{sec}$. A spatial domain of $70 \mathrm{~cm}$ was selected, since this length ensures that the left Lagrangian moving boundary never gets too close to the mixing zone and that the reflected shock off the rigid wall on the right never approaches the mixing zone. In fact, by $819 \mu \mathrm{sec}$ (which corresponds to the end of the experiment), the incident shock has still not reached the far wall nor has the left moving shock caught up to the Lagrangian boundary. Therefore, $70 \mathrm{~cm}$ is a conservative length to use for this problem.

Figure 1 shows a comparison of the mixing zone widths from a coarsely resolved KULL calculation (with 100 zones) to the experimental data. The experimental widths were obtained by examining Schlieren images, while the ones in the KULL calculation were calculated by interpolating the volume fraction profile for $\mathrm{SF}_{6}$ between fraction values of .05 and .95. The agreement is quite good, although there is a slight over- 
prediction at early times and a slight under-prediction at late times. The experimental data is only available between $\sim 300 \mu$ s and $750 \mu$ s.

Figure 2 shows the same data as Figure 1, but also adds the mixing zone widths obtained by interpolating between the $99 \%$ and $1 \%$ values in volume fraction. It is evident that the value of the mixing zone width can vary significantly, depending on the cutoffs used to define it, especially if the volume fraction distribution has long tails. For this reason, we will use the $95 \%$ and $5 \%$ cutoffs to compare to the experimental data (and thus stay away from outliers) and fine-tune the model coefficients, but we will also show the $99 \%$ and $1 \%$ results on occasions where it will prove instructive.

Figure 3 shows a preliminary convergence study using meshes with 100, 200, and 400 zones, where we use the $95 \%$ and $5 \%$ volume fraction cutoffs. The results are somewhat mixed at early times, but at late times it appears as though we may be close to convergence, as the results for the 200 zone and 400 zone run are nearly identical. Figure 4 is identical to Figure 3, except now we use the $99 \%$ and $1 \%$ cutoffs. This figure is more consistent with what one would expect from a convergence study, namely that the widths decrease with increasing resolution (presumably, because the volume fraction profiles are becoming steeper under mesh refinement). The results in this figure indicate that 400 zones may not be sufficient.

Figure 5 shows the $\mathrm{SF}_{6}$ volume fraction profiles at $819 \mu \mathrm{s}$. This figure helps explain why the results from the 100 zone calculation are so scattered. That is, there are only 4 mixed zones at late time in the coarsest calculation, and so we are more subject to interpolation problems. This figure also confirms our suspicions that the 400 zone calculation is not fully converged (at least, we cannot tell without performing higher resolution runs). It is also encouraging that the mixing region is better resolved as the zoning increases, since this means that at some point we should achieve a converged solution.

Running more than 400 zones on a single processor is starting to approach the diminishing returns limit, and so it is worth verifying that parallel runs give the same results as their serial counterparts. In this manner, we can continue to increase the resolution up to 1600 zones or higher. Figures 6 and 7 show pressure profiles and density profiles respectively at $819 \mu \mathrm{s}$. The serial run consisted of 100 zones on a single processor, while the parallel run used 2 processors with 1 processor having 49 onprocessor zones and the other having 51. The results confirm that identical results can be obtained for serial and parallel runs, and also that the python script that manipulates the array variables with various MPI calls is treating the data correctly in both cases.

Figure 8 shows the results of a more comprehensive convergence study where serial runs were performed up to 400 zones, a four processor run was used for the 800 zone calculation, and an eight processor run was employed for the 1600 zone calculation. This figure seems to indicate that "converged" results can be obtained with just 400 zones, since increasing the resolution does not significantly change the mix width. We would reach the same conclusion by examining Figure 9 . Figure 10 shows the density 
profiles at late time for the 3 highest resolution runs. It is interesting that if we focus on the mixing region (which at this time is approximately between $45 \mathrm{~cm}$ and $47 \mathrm{~cm}$, it appears as though 800 zones are necessary for convergence. On the other hand, if we look in the vicinity of the shock front, then we could reasonably argue that we are converging much more slowly. Figure 11 confirms that 800 zones are sufficient to capture the turbulent mixing process for this problem.

Figure 12 shows the time evolution of the turbulent mixing zone for the 200 zone run in which we use a scalar zone centered Q (artificial viscosity) for one run and the CSW (Caramana-Shaskov-Whalen) edge centered Q [6-7] with (on) and without (off) the advection limiter for the other two runs. Since the artificial viscosity will only be felt at the shock front, and as there is no reshock in this problem, the results in Figure 12 should not be too surprising. That is, the mix widths are insensitive to the choice of artificial viscosity used in the KULL calculations. Other physics quantities, like density however, are sensitive, as Figure 13 makes evident. Here, the scalar Q and CSW Q with the limiter turned off give nearly identical results, while there are discernable differences between these runs and the one with the CSW Q with the limiter turned on. Basically, the limiter makes the shock front less diffusive.

Some comments are in order for describing the constant values used in the preceding calculations. The runs used to generate figures 1-13 used the following values for the EZturb model constants and switches:

$\sigma_{\mathrm{M}}=\sigma_{\rho}=.3, \sigma_{\mathrm{U}}=.9, \sigma_{\mathrm{K}}=1, \sigma_{\mathrm{e}}=1.3, \mathrm{c}_{\mu}=.09, \mathrm{c}_{\varepsilon 1}=1.44, \mathrm{c}_{\varepsilon 2}=1.92, \mathrm{c}_{\varepsilon 3}=1.5, \delta_{\text {iso }}=1$, $\delta_{\text {aniso }}=0, \delta_{\mathrm{I}, \mathrm{diss}}=1$

The initial value for the turbulent kinetic energy was chosen to be $1.44 \mathrm{e} 7 \mathrm{~cm}^{2} / \mathrm{s}^{2}$, which is approximately equal to .12 times the shocked air speed squared. Since the initial amplitude was experimentally determined to be $.24 \mathrm{~cm}$, this value was used in conjunction with the kinetic energy to give an initial dissipation rate of $2.28 \mathrm{e} 11 \mathrm{~cm}^{2} / \mathrm{s}^{3}$. Unfortunately, the only value that is "sacrosanct" is $c_{\mu}$. The other constants can be modified within reason. For example, in combustion applications with hydrocarbon gases, Prandtl number around .7 are usually observed. Self-similarity arguments also suggest that all of the $\sigma$ 's, with the exception of $\sigma_{\varepsilon}$ should be equal to each other. With these constraints in mind, as well as the fact that the mix widths change dramatically as we go from 100 zones to 400 zones, we can again attempt to match the data. Figure 14 shows the final result, with the settings now at:

$\sigma_{\mathrm{M}}=\sigma_{\rho}=\sigma_{\mathrm{U}}=\sigma_{\mathrm{K}}=.7, \sigma_{\mathrm{e}}=1.3, \mathrm{c}_{\mu}=.09, \mathrm{c}_{\varepsilon 1}=1.44, \mathrm{c}_{\varepsilon 2}=2.14, \mathrm{c}_{\varepsilon 3}=1.1, \delta_{\text {iso }}=1, \delta_{\text {aniso }}=$ $0, \delta_{\mathrm{I}, \mathrm{diss}}=1$.

\section{References:}

[1] B. Benjamin, D. Besnard, and J.F. Haas, Shock and Reshock of an Unstable Interface, Los Alamos Report LA-UR-92-1185, 1993. 
[2] M. Vetter and V. Sturtevant, Experiments on the RM Instability of an Air/SF6 Interface, Shock Waves, 4, p. 247-252, 1995.

[3] F. Poggi, M.H. Thorembey, and G. Rodriguez, Velocity Measurements in turbulent Gaseous Mixtures induced by RM Instability, Phys. Fluids, 10, Number 11, p. 2698-2700, 1998.

[4] F. Harlow and A. Amsden, Fluid Dynamics - A LASL Monograph, LA-4700, 1971. [5] J. Anderson, Modern Compressible Flow, McGraw-Hill, 1990.

[6] E.J. Caramana, M.J. Shashkov, and P.P. Whalen, Formulations of Artificial Viscosity for Multi-dimensional Shock Wave Computations, JCP, 144, p. 70-97, 1998.

[7] M. Ulitsky, A Tensor Hyperviscosity Model in KULL, UCRL-TR-213310.

\section{Gnuplot}

Mixing Widths (95\% and 5\%) vs. Time for Benjamin

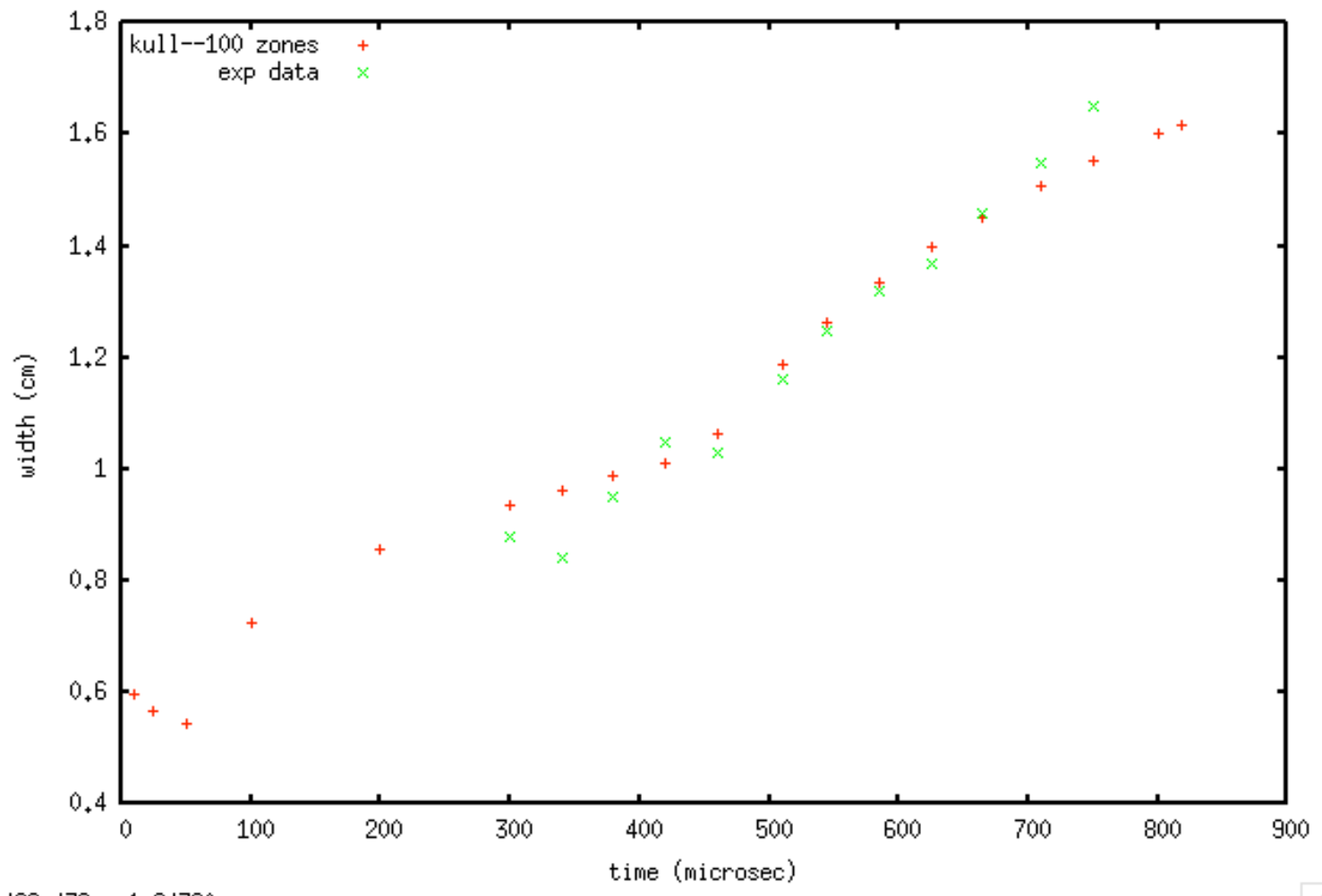

$468,476, \quad 1,94380$

Figure 1 
Mixing Widths vs. Time for Benjamin

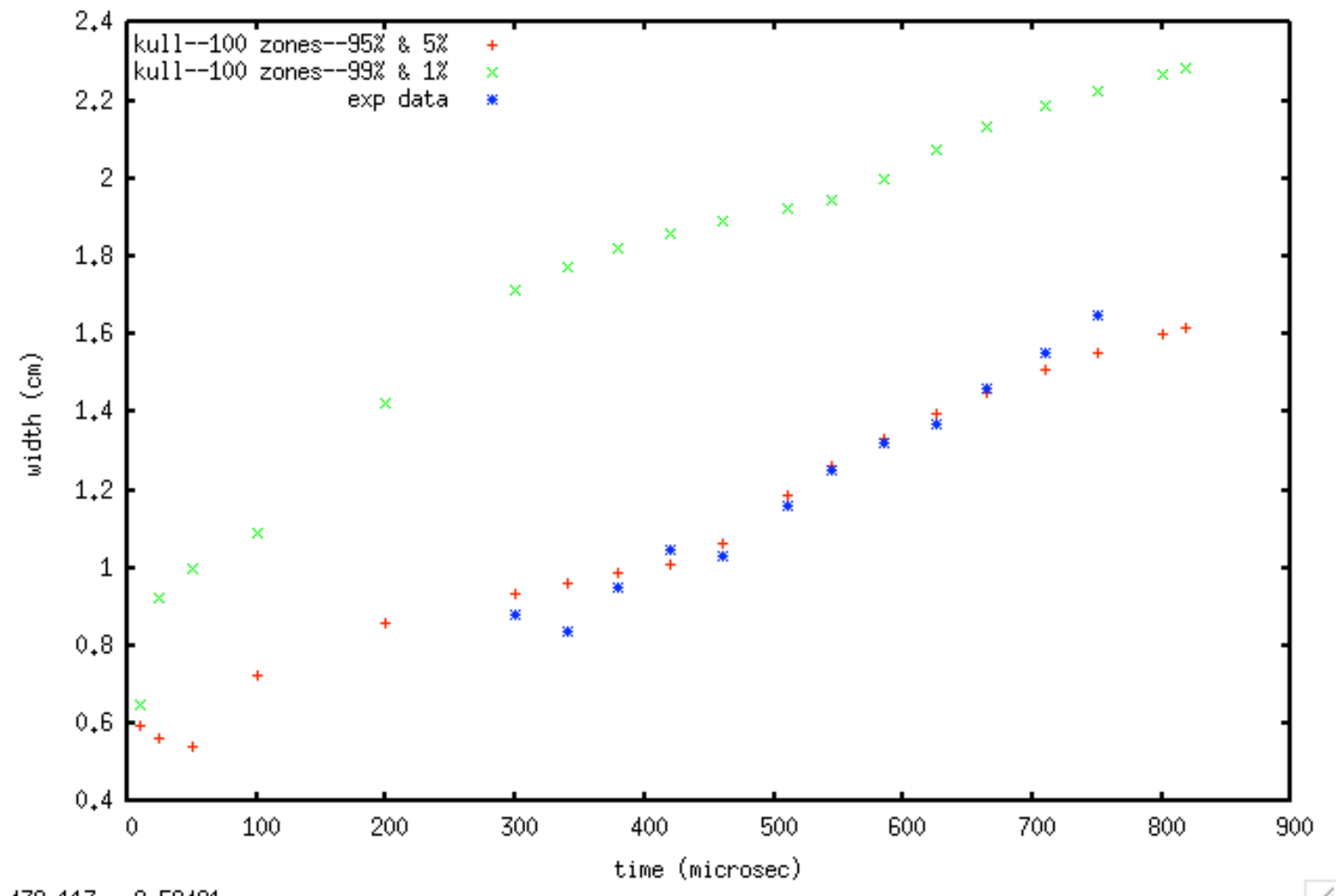

$439.117, \quad 2,59481$

Figure 2 
Mixing Widths vs. Time for Benjamin

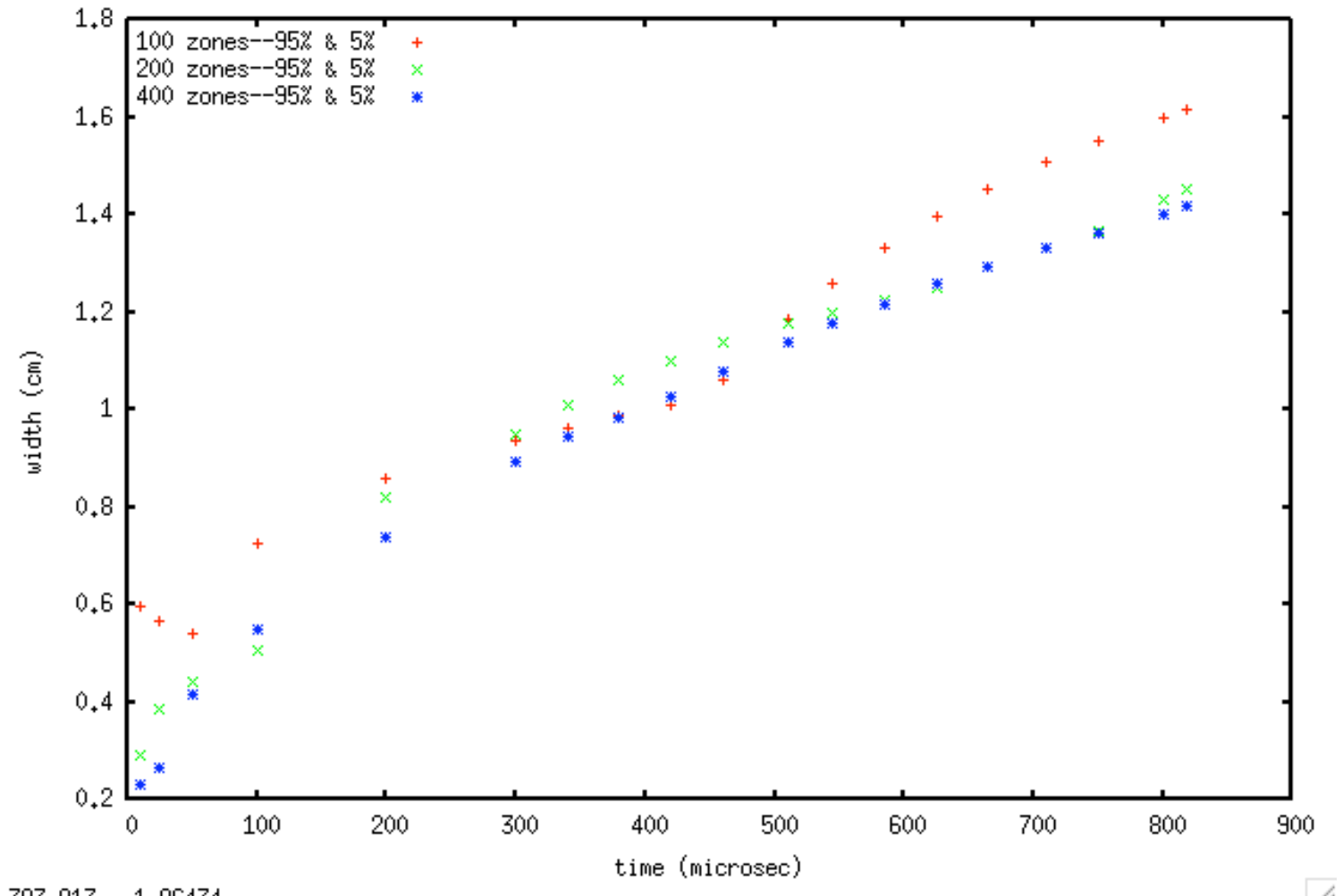

$393,813, \quad 1,96434$

Figure 3 
Mixing Widths vs. Time for Benjamin

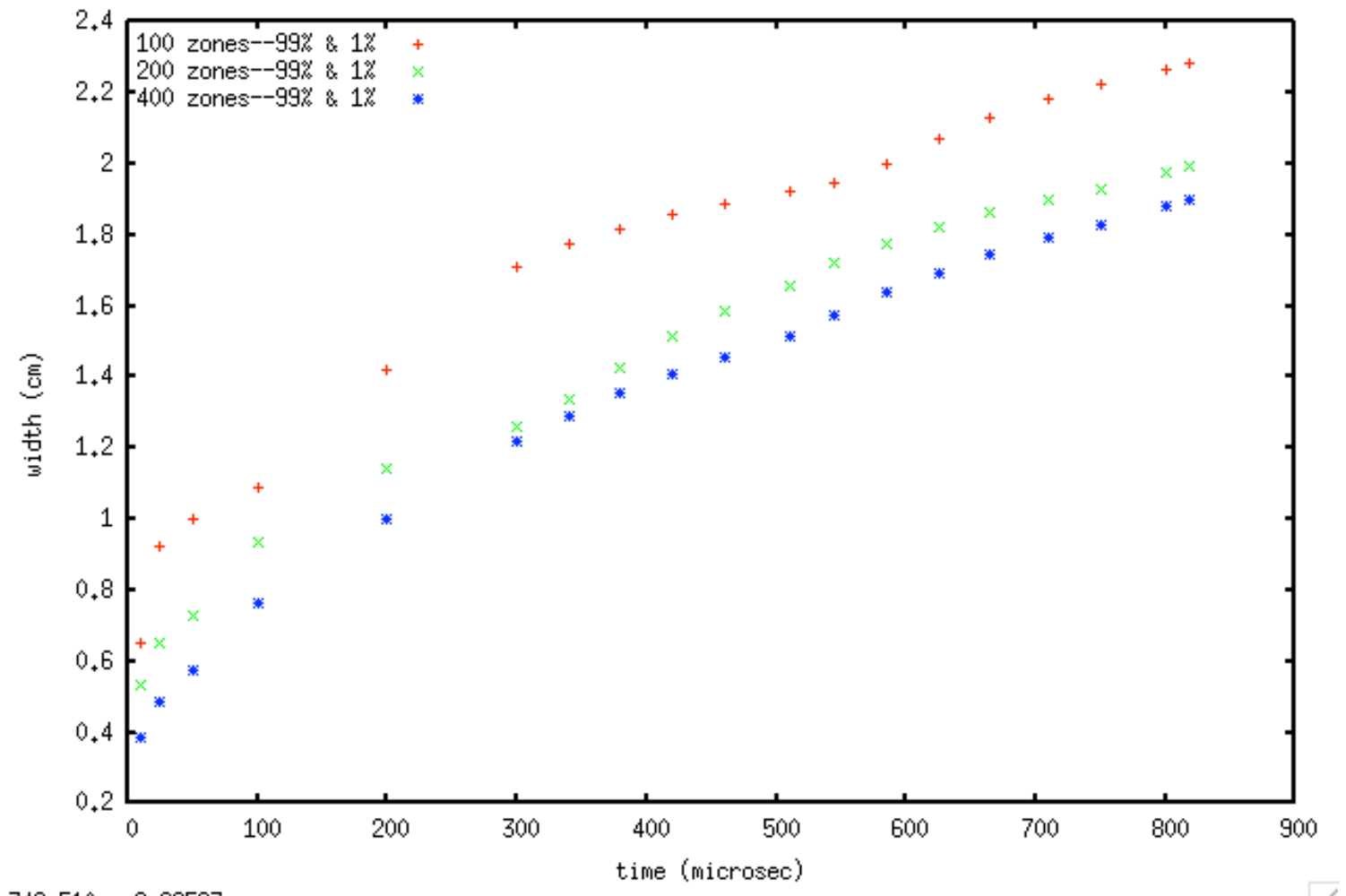

$348,510, \quad 2,62597$

Figure 4 
SF6 Volume Fraction Profiles for Benjamin at 819 microsec

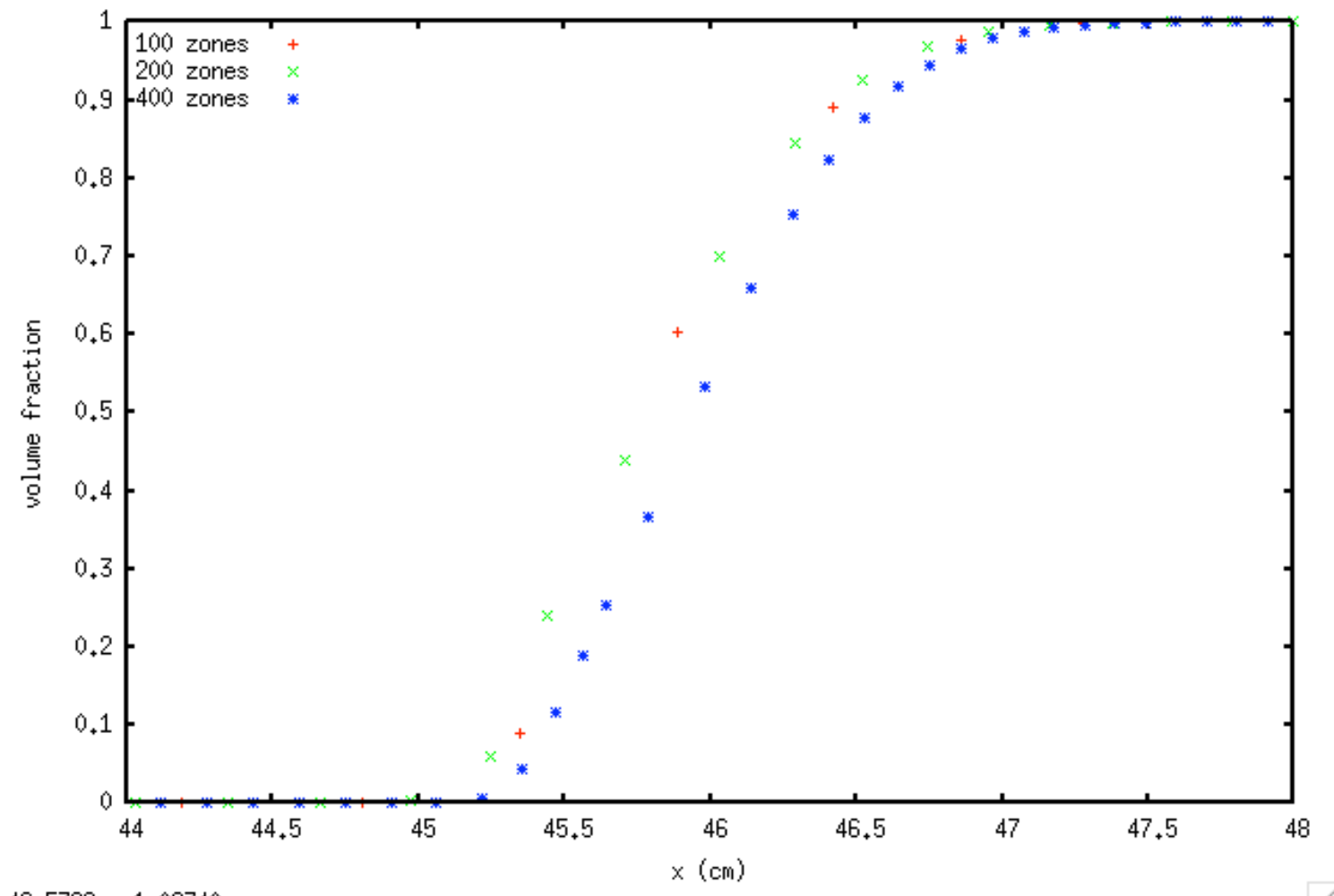

$46,5782, \quad 1,09740$

Figure 5 


\section{$1000 \quad X$ Gnuplot}

Pressure Profiles for Benjamin at 819 microsec

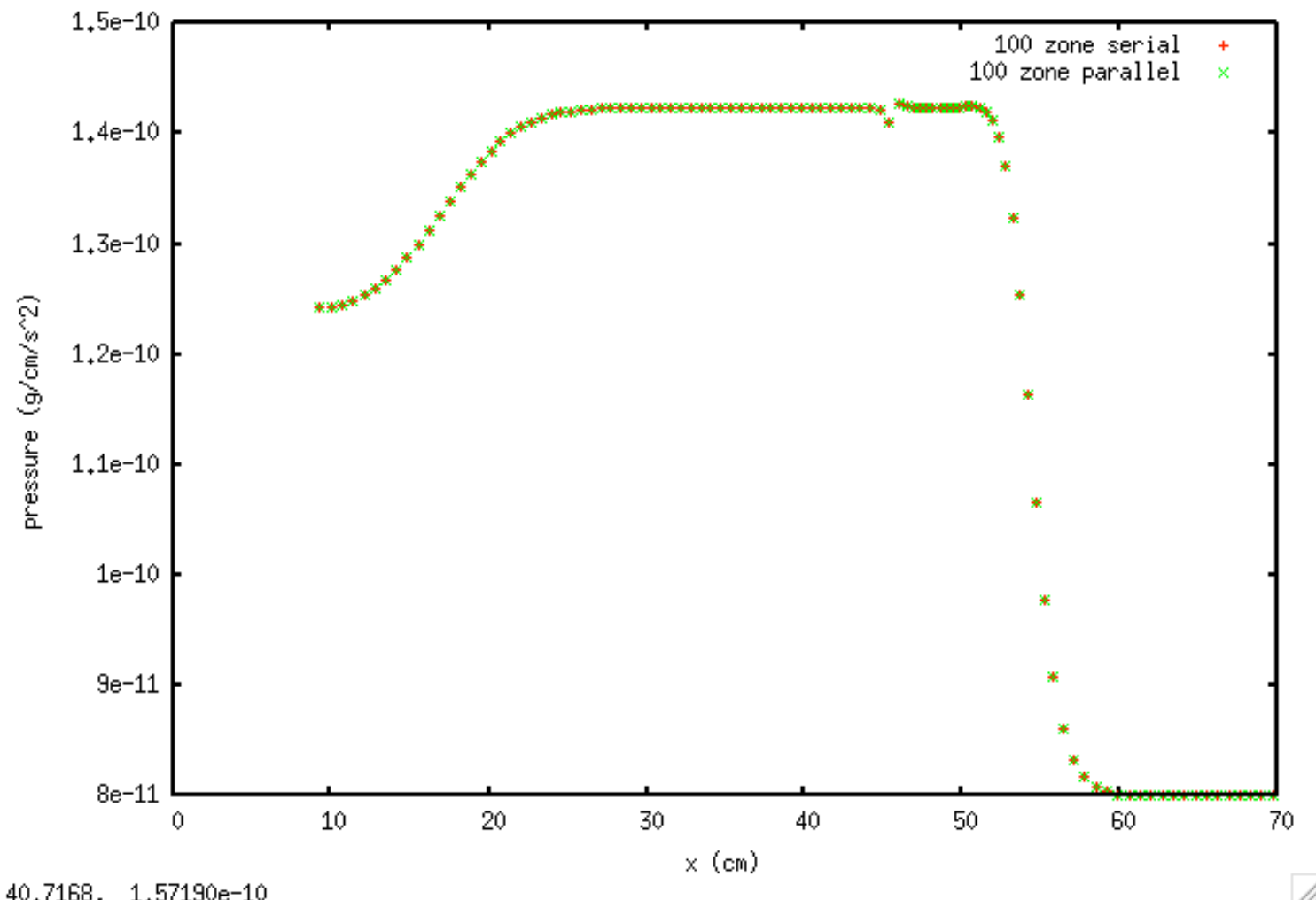

Figure 6 


\section{X Gnuplot}

Density Profiles for Benjamin at 819 microsec

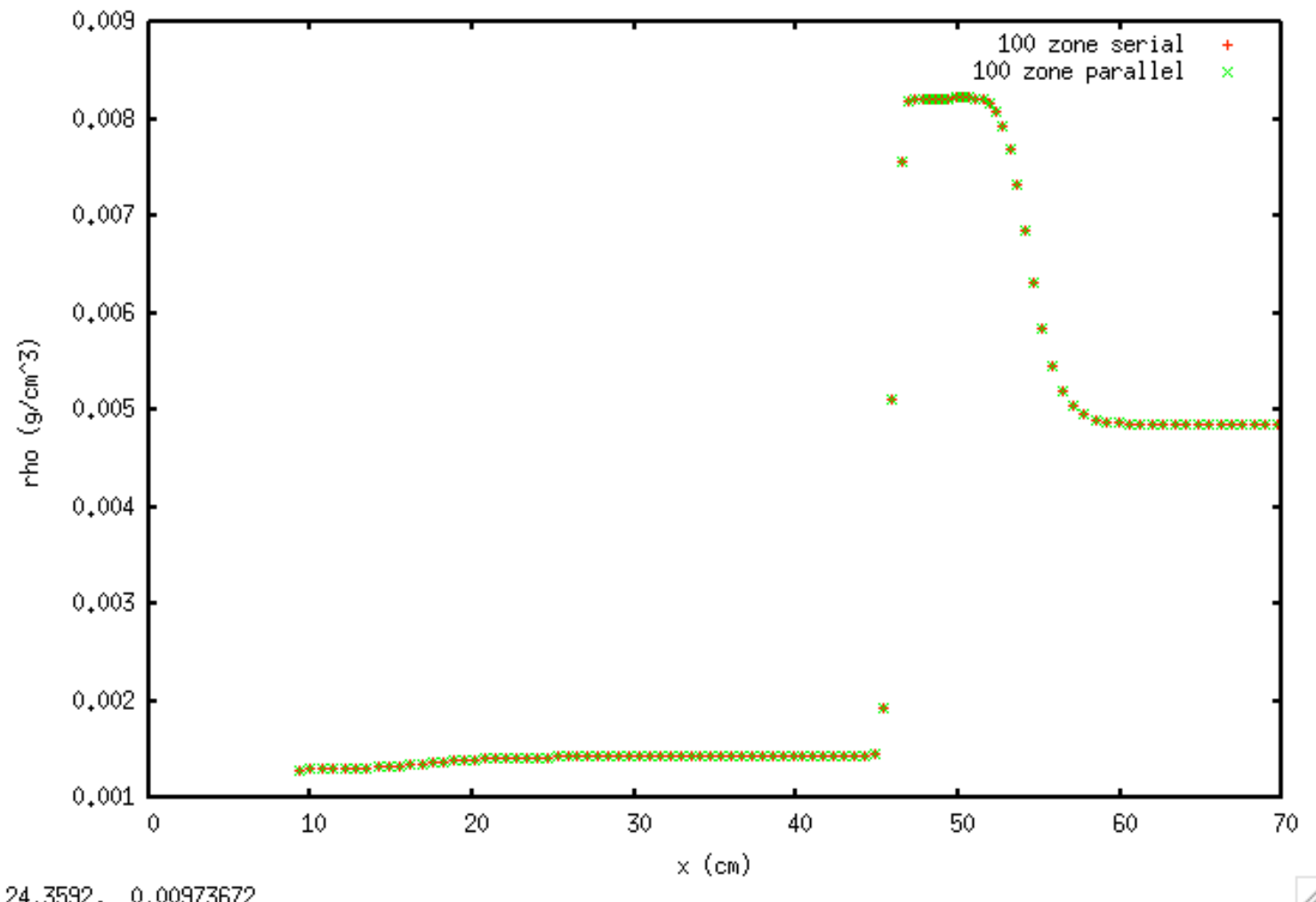

Figure 7 
Mixing Widths (95\% and 5\%) vs. Time for Benjamin

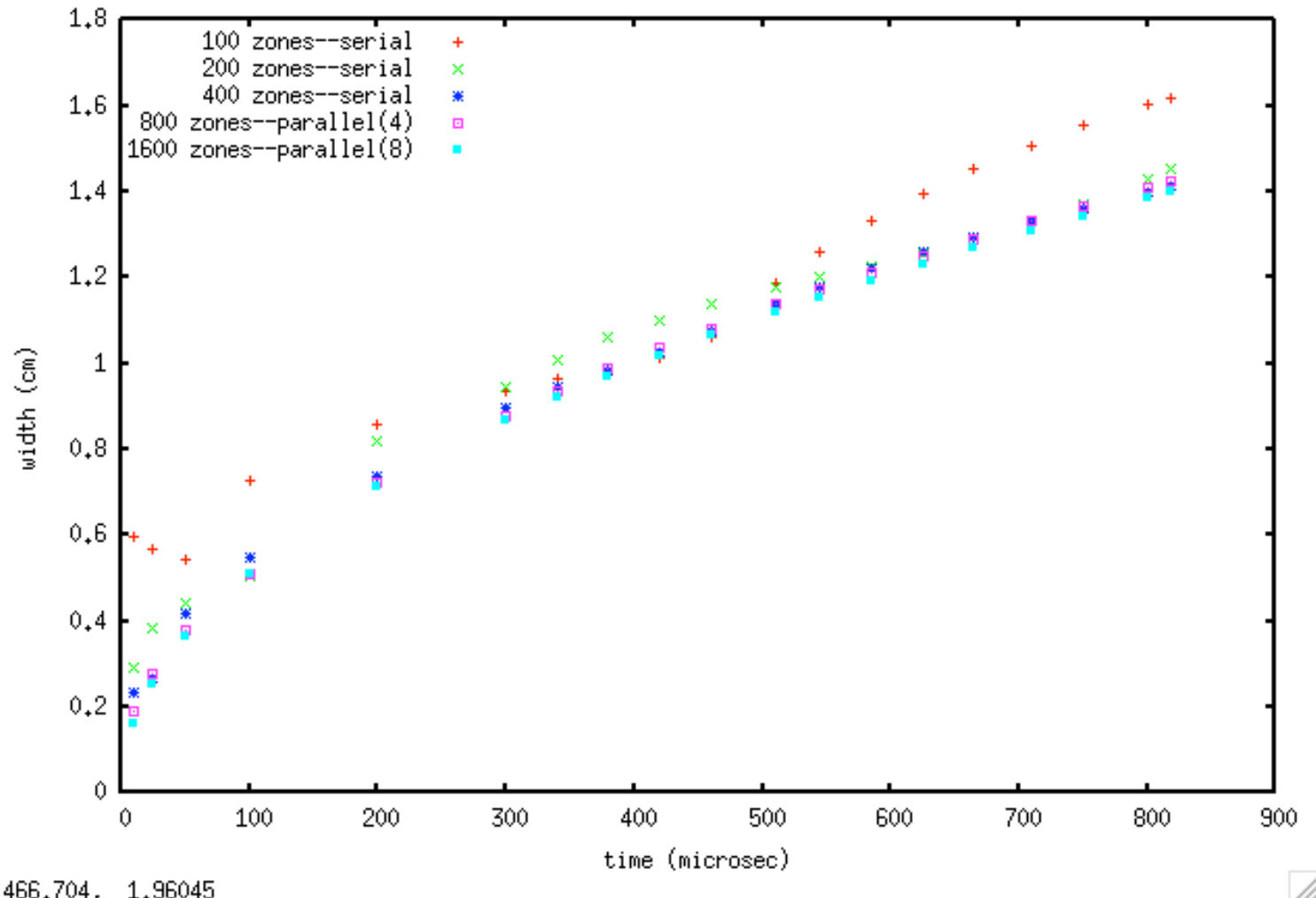

Figure 8 
Mixing Widths (99\% and 1\%) vs. Time for Benjamin

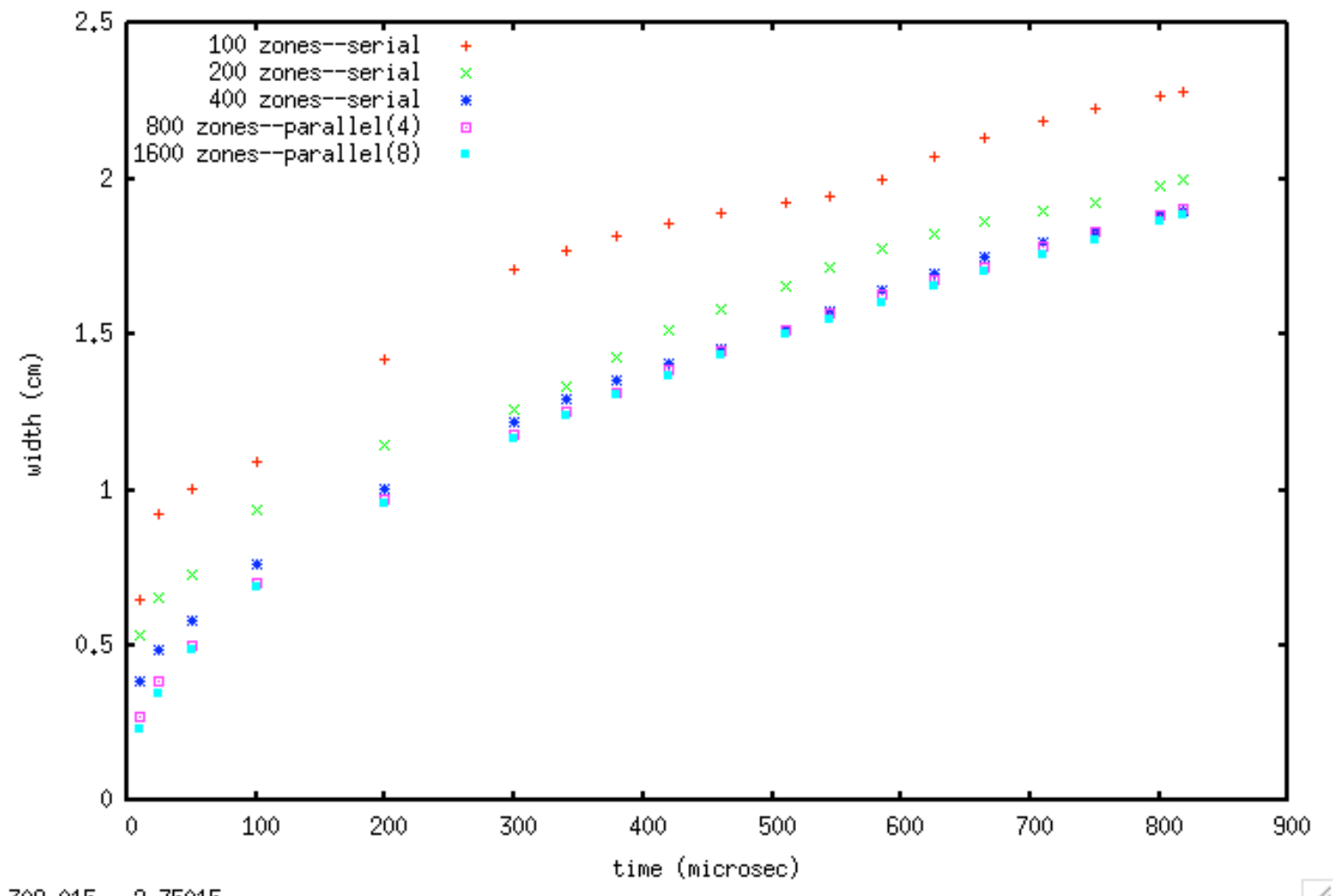

$308,015, \quad 2,75015$

Figure 9 


\section{$000 \quad$ X Gnuplot}

Density Profiles for Benjamin at $t=819$ microsec

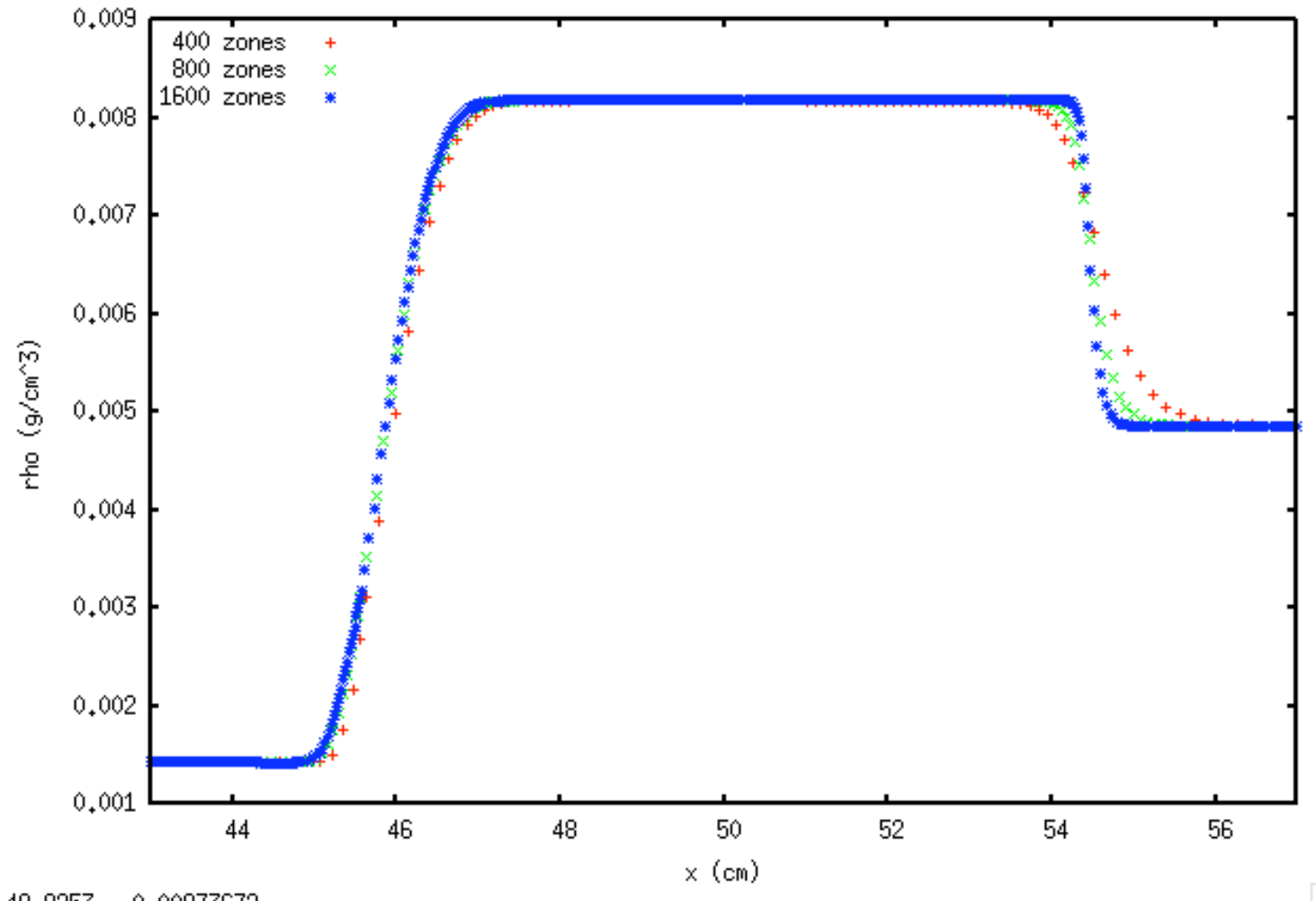

$48,8253, \quad 0,00973672$

Figure 10 


\section{$000 \quad$ X Gnuplot}

SF6 Volume Fraction Profiles for Benjamin at 819 microsec

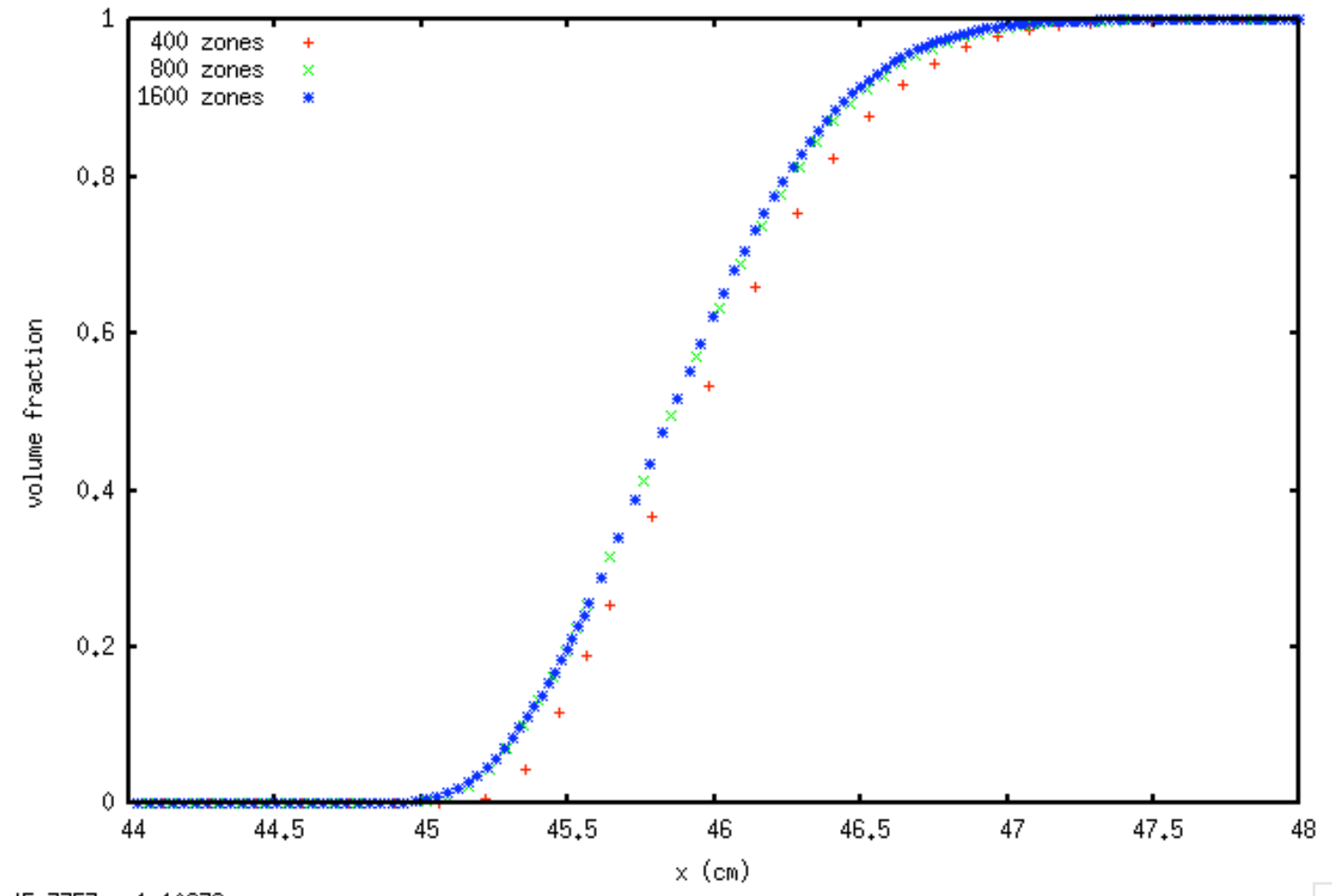

$45,3757,1,10272$

Figure 11 
Mixing Widths (95\% \& 5\%) vs. Time for Benjamin with 200 zones

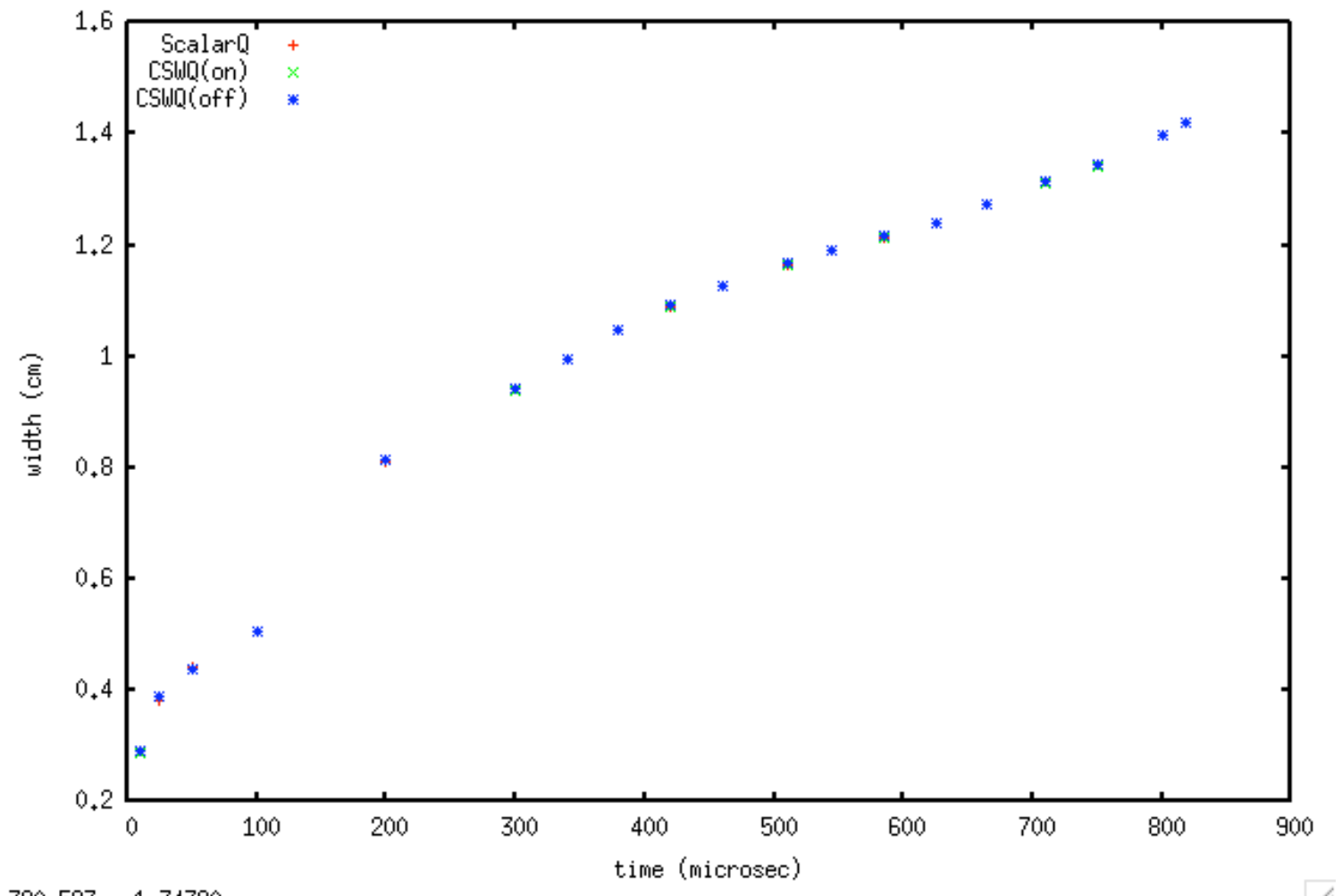

$390,523,1,74380$

Figure 12 
Density Profiles for Benjamin at $t=819$ microsec with 200 zones

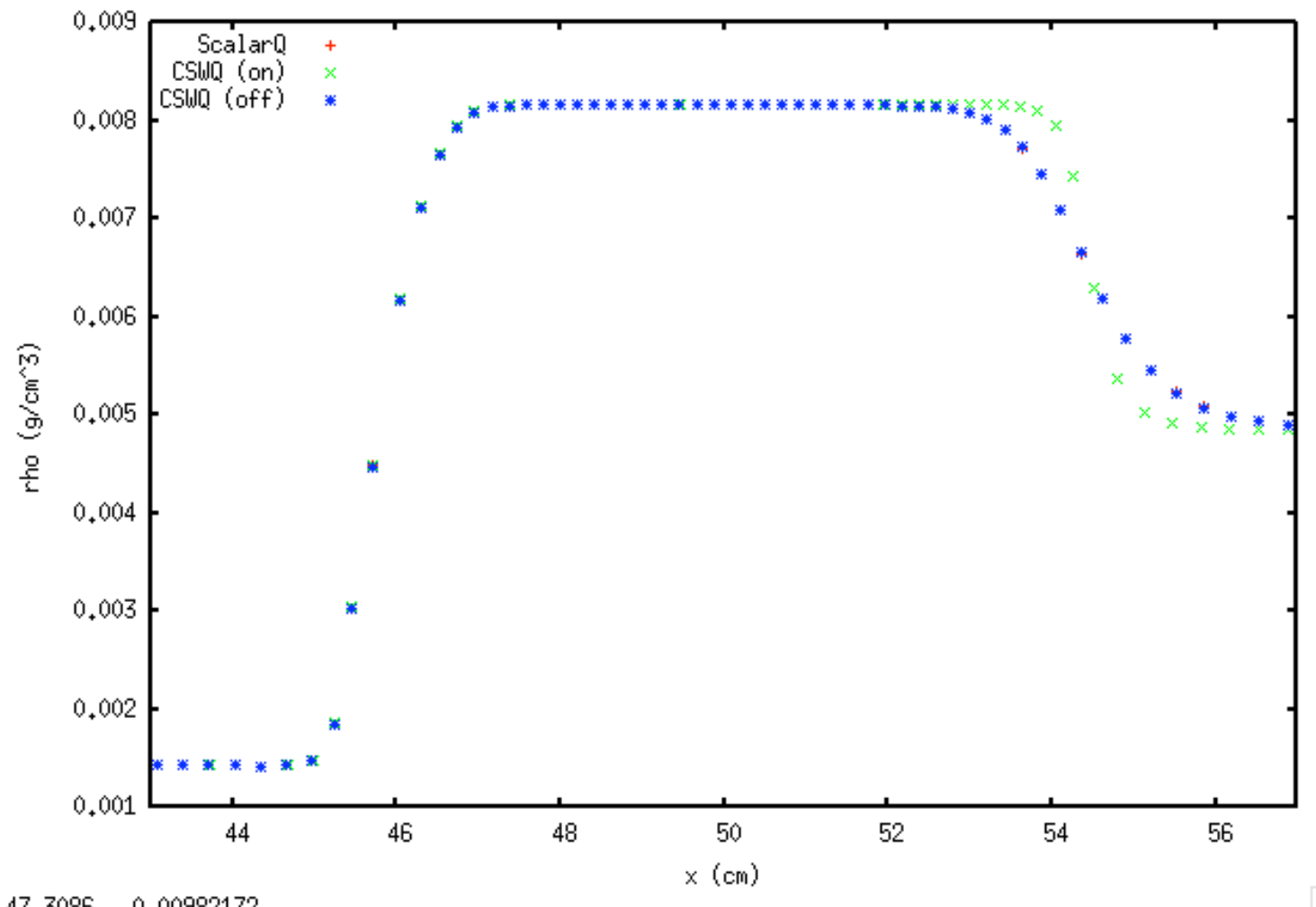

$47,3086, \quad 0.00982172$

Figure 13 
Mixing Widths (95\% and 5\%) vs. Time for Benjamin

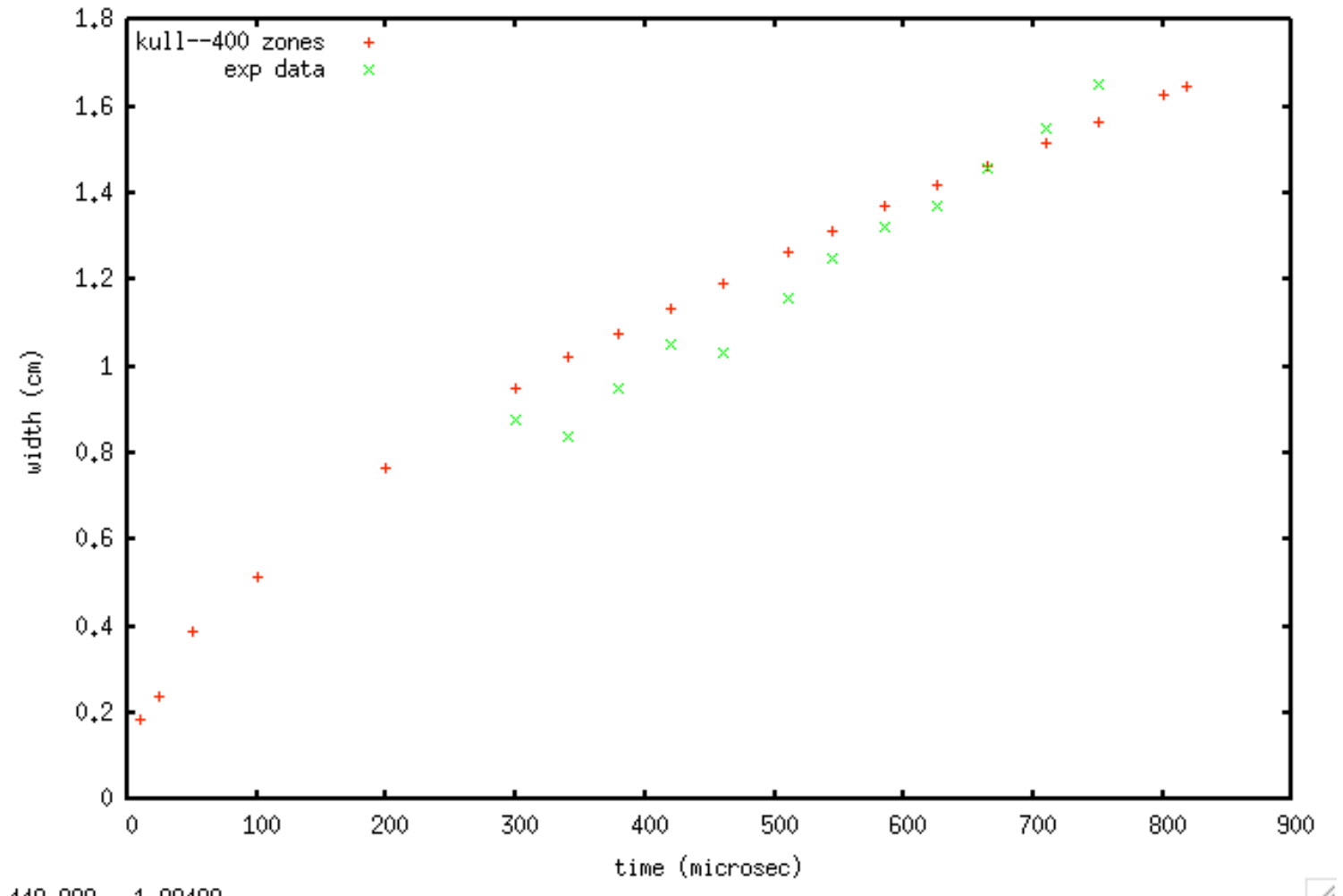

$448,988, \quad 1,98489$

Figure 14 